\title{
Informationskompetenz durch Schulungen und Führungen
}

\author{
Ein Rückblick auf das Jahr 2017
}

Die Landesbibliothek versteht sich als ein modernes Lern- und Informationszentrum, das ihr Schulungs- und Führungsangebot den Bedarfen spezifischer Zielgruppen anpasst. Als "Teaching Library" bietet sie bildungsrelevante Aktivitäten für Schüler, ${ }^{1}$ Studierende, Lehrer und Dozenten, aber auch für die allgemeine, interessierte Öffentlichkeit an. Sie ist kompetente Bildungspartnerin lokaler und regionaler Schulen, Universitäten und Hochschulen und trägt mit ihren Veranstaltungen und Angeboten dazu bei, die Informations- und Medienkompetenz ihrer Nutzer zu stärken. Dabei orientiert sich die Praxis und Planung der WLB verstärkt am zuletzt verabschiedeten "Referenzrahmen Informationskompetenz" (2016) des Bundesvorstands des Deutschen Bibliotheksverbandes e.V. (dbv). ${ }^{2}$ Der Informationskompetenz werden fünf Teilkompetenzen auf sechs Niveaustufen von der Grundschule bis zur akademischen Bildung zugeschrieben: Das "Suchen", „Prüfen“, die Schaffung bzw. Aneignung von "Wissen" sowie die Kompetenzen "Darstellen" und „Weitergeben".

Der Schwerpunkt der insgesamt 247 Veranstaltungen zur Vermittlung von Informationskompetenz lag 2017 wie in den Vorjahren auf dem Kursangebot für Schulen:
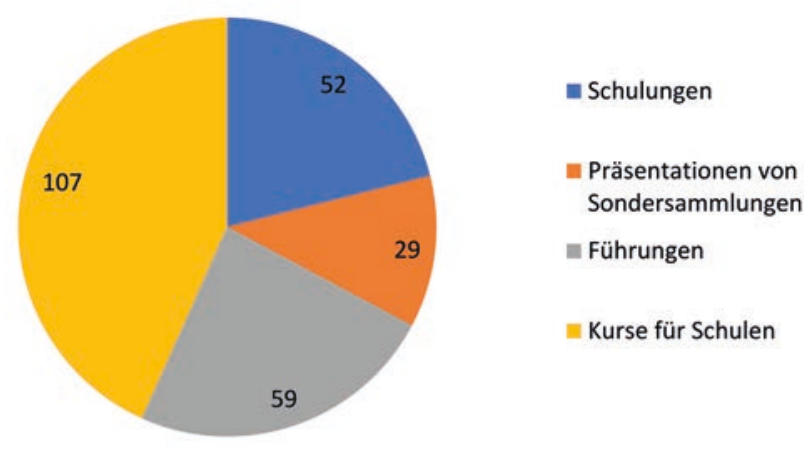

Eurse für Schulen
Die 107 Kurse für Schulen, die im Jahr 2017 stattgefunden haben, setzen sich mehrheitlich aus 98 Veranstaltungen „Fit für Seminarkurse" zusammen, einem Angebot für Schüler der gymnasialen Oberstufe, das jedoch auch von Berufsschulen und Oberstufenkollegs wahrgenommen wird. Im Fokus steht hier die Teilkompetenz des Suchens. In einem ersten Schritt werden die Lernenden über die Literaturangebote der Bibliothek im Präsenzbestand des Hauptlesesaals und über die ausleinbaren Bestände des Magazins informiert. Im zweiten Schritt geht es darum, den Wissensbedarf mit geeigneten Suchbegriffen zu formulieren. Anschließend werden anhand von Beispielen die wichtigsten Suchstrategien im WLB-Katalog und einer Datenbank vermittelt. Das Üben der thematischen und formalen Suche nach Büchern, Aufsätzen und Zeitungsartikeln soll den Schülern dazu verhelfen, künftig selbstständig Literatur zu finden und auszuwählen. Das Prüfen der Quelle nach wissenschaftlichen Kriterien spielt auch eine wichtige Rolle.

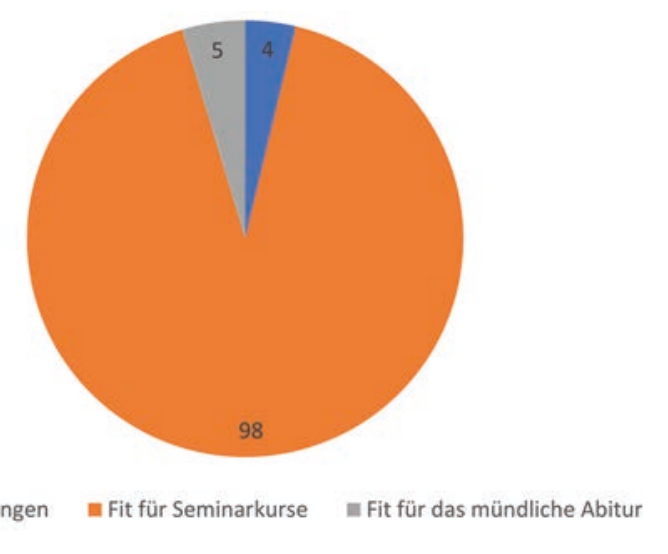

Abb. 2: Zusammensetzung und Verteilung der Kurse für Schulen im Jahr 2017
1) Zur besseren Lesbarkeit wird im vorliegenden Artikel das generische Maskulinum verwendet. Es bezieht sich gleichermaßen auf weibliche und männliche Personen.

2) Klingeberg, Andreas: "Referenzrahmen Informationskompetenz". In: http://www.bibliotheksverband.de/fileadmin/user_upload/Kommissionen/Kom_Infokompetenz/2016_11_neu_Referenzrahmen-Informationskompetenz_endg_2_Kbg.pdf (Zugang: 01.03.2018). Siehe auch: http:// www.informationskompetenz.de/index.php/referenzrahmen/ (Zugang: 01.03.2018). 
Das Format „Fit für das mündliche Abitur" ist ähnlich konzipiert und richtet sich ebenfalls an die gymnasiale Oberstufe. Die Veranstaltung "Fit für Führungen" bildet Lehrkräfte fort, die die Informationsressourcen der Bibliothek im Hinblick auf die eigene Unterrichtsvorbereitung besser kennenlernen und als Multiplikatoren agieren möchten. Das weitere heterogene Schulungsangebot der WLB und seine Frequentierung lassen sich in vier Kategorien darstellen:

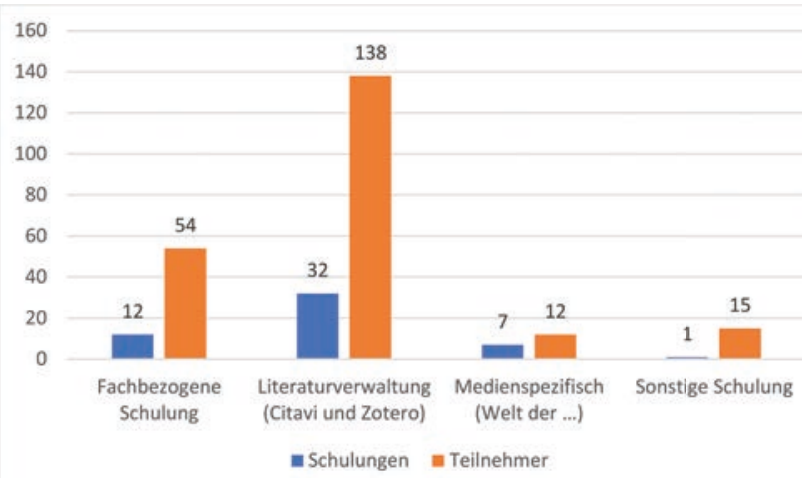

Abb. 3: Verteilung des heterogenen Schulungsangebots im Jahr 2017

Neben den fachbezogenen Schulungen, die in erster Linie von Studierendengruppen der jeweiligen Fachwissenschaften im Rahmen von Propädeutika und anderen akademischen Seminaren wahrgenommen werden, unterstützen unsere fachübergreifenden Schulungen die interessierten Nutzer beim Umgang mit Programmen zur Literaturverwaltung (Citavi und Zotero) sowie bei der spezifischen Recherche und Evaluation unterschiedlicher Medien. Hierfür stehen die Formate "Welt der Bücher", „Welt der Aufsätze“, „Welt der Zeitungen“. Bei allen fachbezogenen und fachübergreifenden Schulungen geht es um die Aneignung von Kompetenzen im Umgang mit gedruckten sowie elektronischen Medien, relevanten Bibliothekskatalogen, Katalogverbünden, elektronischen Datenbanken und Fachportalen im Internet. Anhand von praktischen Beispielen werden die unterschiedlichen Recherchemöglichkeiten erläutert und Suchstrategien vertieft.

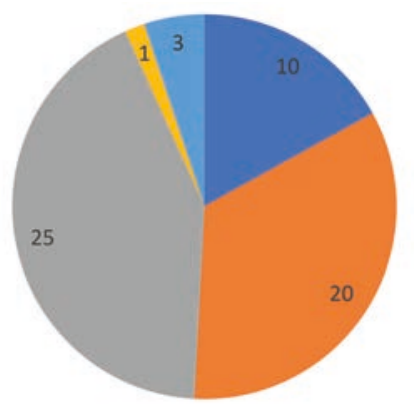

= Mittwochsführung = Gruppenführung = Fachbezogene Führung
$=$ Fremdsprachige Führung $=$ Sonstige Führung

Abb. 4: Verteilung der Führungen im Jahr 2017

Im Bereich der Führungen wurden insbesondere die Gruppenführungen mit 331 Teilnehmern und die fachbezogenen Führungen mit 422 Teilnehmern von insgesamt 827 teilnehmenden Nutzern bevorzugt.

Die allgemeinen Mittwochs- und Gruppenführungen bieten einen Rundgang durch das Haus, eine kurze Einführung in die Geschichte und Aufgabenschwerpunkte des Hauses sowie in die zentralen Servicebereiche. Das Ziel ist es, Erstbenutzern und interessierten Personen umfassende Grundlagen zur Benutzung der Bibliothek zu vermitteln.

Bei den fachbezogenen Führungen lernen unsere Nutzer die Bibliothek und ihre digitalen Angebote aus der Sicht Ihres Faches kennen. Im Fokus stehen neben der Benutzung der Bibliothek der fachspezifische Lesesaalbestand sowie die Literatursuche im WLB-Katalog und in fachlich relevante Datenbanken.

Hinter den sonstigen Führungen verbargen sich im Jahr 2017 Besuche interessierter Gäste wie der Buchpaten in der Restaurierungs- und Digitalisierungswerkstatt der WLB.

Insgesamt weist das Jahr 2017 im Vergleich zu 2016 einen leichten Rückgang der Veranstaltungszahlen (von 255 auf 247 Veranstaltungen), zugleich einen leichten Anstieg der Gesamtdauer der Veranstaltungen (von 365 auf 368 Stunden) und der Teilnehmerzahlen (von 2962 auf 2980) auf. 